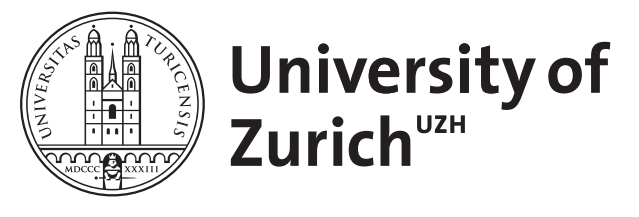

\title{
Primärer Hyperaldosteronismus bei Katzen
}

Willi, Barbara ; Kook, Peter H ; Quante, S ; Boretti, Felicitas S ; Sieber-Ruckstuhl, Nadja S ; Grest, Paula ; Scherrer, O ; Riond, Barbara ; Hofmann-Lehmann, Regina ; Nussberger, J ; Reusch, Claudia E

\begin{abstract}
Primary hyperaldosteronism is a clinical syndrome characterized by an elevated aldosterone secretion by the adrenals. The present case series describes 7 cats with primary hyperaldosteronism, which were presented between 2002 and 2011. Common clinical symptoms were weakness, anorexia, cervical ventroflexion and blindness. All cats showed hypokalemia. In 6 cats, blood pressure was determined: 5 cats showed hypertension, of which 4 animals exhibited retinal detachment and blindness. In the ultrasonographic examination, unilateral adrenomegaly was present in 6 cats whereas one animal showed normal adrenals. In 4 cats, the serum aldosterone concentration was above the reference range. Five cats underwent unilateral adrenalectomy, which was accomplished uneventfully and returned the electrolytes back to normal. Histopathological examination of the adrenals revealed 2 carcinomas and 4 adenomas; one cat with ultrasonographic normal adrenals exhibited bilateral nodular hyperplasia.
\end{abstract}

DOI: https://doi.org/10.1024/0036-7281/a000402

Other titles: Primary hyperaldosteronism in cats: a series of seven cases

Posted at the Zurich Open Repository and Archive, University of Zurich ZORA URL: https://doi.org/10.5167/uzh-68475

Journal Article

Accepted Version

Originally published at:

Willi, Barbara; Kook, Peter H; Quante, S; Boretti, Felicitas S; Sieber-Ruckstuhl, Nadja S; Grest, Paula; Scherrer, O; Riond, Barbara; Hofmann-Lehmann, Regina; Nussberger, J; Reusch, Claudia E (2012). Primärer Hyperaldosteronismus bei Katzen. Schweizer Archiv für Tierheilkunde, 154(12):529-537.

DOI: https://doi.org/10.1024/0036-7281/a000402 


\section{Primärer Hyperaldosteronismus bei Katzen: eine Fallserie}

2 B. Willi ${ }^{1,2}$, P.H. Kook ${ }^{1}$, S. Quante ${ }^{1}$, F.S. Boretti ${ }^{1}$, N.S. Sieber-Ruckstuhl ${ }^{1}$, P. Grest ${ }^{3}$, O.

3 Scherrer $^{4}$, B. Riond ${ }^{2}$, R. Hofmann-Lehmann ${ }^{2}$, J. Nussberger ${ }^{5}$, C.E. Reusch ${ }^{1}$

5 ' ${ }^{1}$ Klinik für Kleintiermedizin, ${ }^{2}$ Veterinärmedizinisches Labor und ${ }^{3}$ Institut für 6 Veterinärpathologie der Universität Zürich, ${ }^{4}$ Kleintierpraxis Lindengarten, Kloten, $7{ }^{5}$ Département de médecine interne, Centre Hospitalier Universitaire Vaudois, 8 Lausanne, Schweiz.

\section{Zusammenfassung}

11 Der Begriff primärer Hyperaldosteronismus beschreibt ein Krankheitsbild, welches durch eine erhöhte Aldosteronsekretion durch die Nebennieren charakterisiert ist. Diese Fallserie beschreibt 7 Katzen mit primärem Hyperaldosteronismus, welche zwischen 2002 und 2011 vorgestellt wurden. Die häufigsten klinischen Symptome waren Schwäche, Anorexie, zervikale Ventroflexion und Blindheit. Bei allen Katzen lag eine Hypokaliämie vor. Bei 6 Katzen wurde der Blutdruck bestimmt: 5 Katzen zeigten eine Hypertonie, welche bei 4 Tieren mit einer Retinaablösung und Blindheit einherging. Im Ultraschall wurde bei 6 Katzen eine unilaterale Adrenomegalie festgestellt, bei 1 Katze waren die Nebennieren ultrasonographisch normal. Vier Katzen wiesen eine erhöhte Aldosteron-Konzentration im Serum auf. Bei 5 Katzen wurde eine Adrenalektomie durchgeführt, die komplikationslos verlief und zur Normalisierung der Elektrolytwerte führte. Die histologische Untersuchung der Nebennieren ergab 2 Karzinome und 4 Adenome; bei einer Katze mit ultrasonographisch normalen Nebennieren wurde eine bilaterale knotige Hyperplasie festgestellt.

Schlüsselwörter: Primärer Hyperaldosteronismus, Katze, Hypertonie, Hypokaliämie, Aldosteron

\section{Summary}

Primary hyperaldosteronism is a clinical syndrome characterized by an elevated aldosterone secretion by the adrenals. The present case series describes 7 cats with primary hyperaldosteronism, which were presented between 2002 and 2011.

32 Common clinical symptoms were weakness, anorexia, cervical ventroflexion and 33 blindness. All cats showed hypokalemia. In 6 cats, blood pressure was determined: 5 
cats showed hypertension, of which 4 animals exhibited retinal detachment and blindness. In the ultrasonographic examination, unilateral adrenomegaly was present in 6 cats whereas one animal showed normal adrenals. In 4 cats, the serum aldosterone concentration was above the reference range. Five cats underwent unilateral adrenalectomy, which was accomplished uneventfully and returned the electrolytes back to normal. Histopathological examination of the adrenals revealed 2 carcinomas and 4 adenomas; one cat with ultrasonographic normal adrenals exhibited bilateral nodular hyperplasia.

Key words: primary hyperaldosteronism, cat, hypertension, hypokalemia, aldosterone

\section{Einleitung}

45 Unter dem Begriff primärer Hyperaldosteronismus wird ein Krankheitsbild verstanden, das durch eine erhöhte Aldosteronsekretion durch die Nebennierenrinde gekennzeichnet ist. Bei Katzen wurde diese Erkrankung erstmals 1983 dokumentiert und seither wurden 36 Fälle beschrieben (Eger et al., 1983, Flood et al., 1999, Mackay et al., 1999, Moore et al., 2000, Rijnberk et al., 2001, Ash et al., 2005, DeClue et al., 2005, Javadi et al., 2005, Reimer et al., 2005, Rose et al., 2007, Djajadiningrat-Laanen et al., 2008, Briscoe et al., 2009, Renschler and Dean, 2009). Die Ursache sind neoplastische oder hyperplastische Veränderungen der Nebennierenrinde, die zu einer autonom erhöhten Aldosteronsekretion führen, das heißt die Sekretion findet unabhängig von der Höhe der Reninkonzentration statt (Fig. 1; Schulman, 2010). Aldosteron erhöht die Kaliumsekretion in die Nierentubuli und die Rückresorption von Natrium und Wasser im distalen Nephron. Der erhöhte Kaliumverlust über den Urin und die daraus resultierende Hypokaliämie äussern sich klinisch vor allem als Muskelschwäche. Daneben zeigen diese Katzen eine ausgeprägte Hypertonie, die durch das erhöhte intravaskuläre Volumen und direkte Effekte von Aldosteron auf die Blutgefässe erklärt wird (Schulman, 2010). Die Hypertonie führt zu Endorganschädigungen, insbesondere im Auge (intraokuläre Blutung, hypertensive Retinopathie), und Katzen mit primärem

\section{4} Hyperaldosteronismus werden oft mit Retinablutung, Retinaablösung oder

65 Die Diagnose kann mittels bildgebender Verfahren und Hormonmessungen gestellt werden. In den meisten Fällen weisen die Patienten Form- und 
67 Grössenveränderungen der Nebennieren auf, die mittels Ultraschall dargestellt 68 werden können (Schulman, 2010). Der Nachweis einer erhöhten Aldosteron69 Konzentration im Blut ist charakteristisch für einen primären Hyperaldosteronismus 70 (Schulman, 2010). Es gilt jedoch zu beachten, dass eine hohe Aldosteron71 Konzentration auch bei dehydrierten Tieren vorkommt, als physiologische Reaktion 72 auf einen Blutvolumenmangel. Eine bessere Aussage ist mit einer gleichzeitigen 73 Messung der Reninaktivität im Blut und der Berechnung eines Quotienten 74 (Aldosteronkonzentration:Reninaktivität) möglich: bei Katzen mit primärem 75 Hyperaldosteronismus ist dieser Quotient höher als bei gesunden Katzen (Javadi et 76 al., 2005).

77 Ziel der vorliegenden Studie war die Beschreibung der klinischen Präsentation, der 78 Laborveränderungen, der Therapie und des Krankheitsverlaufes bei 7 Katzen mit 79 primärem Hyperaldosteronismus. Außerdem werden verschiedene diagnostische 80 Tests diskutiert.

\section{Material und Methoden}

82 Tiere

83 Es wurden 7 Katzen mit primärem Hyperaldosteronismus in die retrospektive Studie 84 eingeschlossen (Tab. 1): 6 Katzen waren zwischen 2002 und 2011 an der Klinik für 85 Kleintiermedizin der Universität Zürich, eine Katze 2008 in einer Tierarztpraxis in der 86

Die hämatologische und

blutchemische Untersuchung wurde im 93 Veterinärmedizinischen Labor der Universität Zürich durchgeführt. Für die hämatologische Untersuchung wurde ein Cell-Cyn 3500 System (Abbott, Baar, Schweiz) oder Sysmex XT-2000iV (Sysmex Corporation, Kobe, Japan), für die blutchemische ein Cobas Integra 700 System (Roche Diagnostics, Rotkreuz, Schweiz) verwendet.

8 Ultrasonographische Untersuchung des Abdomens 
99 Die ultrasonographische Untersuchung wurde bei 6 Katzen durch Radiologen der 100 Abteilung für bildgebende Diagnostik der Vetsuisse-Fakultät, Universität Zürich, und 101 bei 1 Katze durch einen externen Radiologen durchgeführt. Für die Beurteilung der 102 Grösse der Nebennieren wurden die für Katzen etablierten Referenzwerte verwendet 103 (Zimmer et al., 2000).

\section{Ophtalmologische Untersuchung}

105 Fünf Katzen erhielten eine ophthalmologische Untersuchung mittels Spaltlampe 106 (Kowa SL 14; Kowa Company Ltd., Tokio, Japan) und indirekter Ophtalmoskopie 107 (Heine Omega 500, Heine Optotechnik, Herrsching, Deutschland) durch die 108 Abteilung für Ophtalmologie der Vetsuisse-Fakultät, Universität Zürich. Die Diagnose 109 Retinaablösung wurde gestellt, wenn die Untersuchung des Fundus eine komplette 110 Ablösung oder auch nur einzelne oder multiple bullöse oder flache Abhebungen der 111 Retina zeigte.

\section{Blutdruckmessung}

113 Bei 6 Katzen wurde eine Blutdruckmessung mittels Doppler (Model 811-B; Parks 114 Medical Electonics, Aloha, USA) durchgeführt wie beschrieben (Reusch et al., 2010). 115 Ein systolischer Blutdruck $\geq 150 \mathrm{mmHg}$ wurde als Hypertonie definiert (Brown et al., 116 2007).

\section{Endokrinologische Untersuchungen}

118 Bei allen Katzen wurde die Aldosteron-Konzentration im Serum mittels direktem 119 Radioimmunoassay (RIA, Coat-A-Count Aldosterone, Siemens Medical Solutions 120 Diagnostics, Los Angeles, USA) in einem kommerziellen Labor (Unilabs Dr. Weber, 121 St. Gallen, Schweiz) bestimmt. Es wurden die für Katzen etablierten Referenzwerte 122 verwendet (Zimmer et al., 2000). Bei einer Katze (Nr. 6) wurde zusätzlich das 123 Aldosteron:Renin Verhältnis gemessen und ein Fludrocortison-Suppressionstest 124 durchgeführt. Die Reninaktivität wurde mittels enzymatischem Assay am Centre 125 Hospitalier Universitaire Vaudois in Lausanne bestimmt (Nussberger et al., 1987); da keine Referenzwerte für Katzen vorlagen wurde zum Vergleich die Reninaktivität von

1273 gesunden Katzen mitbestimmt. Dafür wurde $1 \mathrm{ml}$ Blut in ein vorgekühltes EDTA128 Röhrchen verbracht, bei $4^{\circ} \mathrm{C}$ zentrifugiert, das Plasma bis zum Versand bei $-20^{\circ} \mathrm{C}$ 129 gelagert und die Proben auf Trockeneis versandt. In den gleichen Blutproben wurde 130 die Aldosteron-Konzentration mittels Flüssigphasen-Assay bestimmt (Nussberger et 131 al., 1984) und der Quotient Aldosteron-Konzentration (in pmol/l):Reninaktivität (in $132 \mathrm{fmol} / \mathrm{l} / \mathrm{s}$ ) berechnet (Javadi et al., 2004). Für den Fludrocortison-Suppressionstest 
133 wurde Fludrocortison Acetat (Florinef, $0.05 \mathrm{mg} / \mathrm{kg}$ ) über vier Tage 2 x täglich 134 verabreicht und das Aldosteron:Kreatinin Verhältnis im Urin vor und 12 Stunden nach 135 der letzten Verabreichung gemessen (Unilabs Dr. Weber, St. Gallen, Schweiz). Der 136 Urin wurde mittels Zystozentese entnommen und gekühlt versandt (Versandbehälter 137 für Kühltransporte, Sarstedt, Sevelen, Schweiz). Die Aldosteron-Konzentration im 138 Urin wurde mittels direktem RIA (Coat-A-Count Aldosterone, Siemens Medical 139 Solutions Diagnostics, Los Angeles, USA), die Kreatinin-Konzentration mittels Jaffé140 Methode bestimmt (ARCHITECT® c Systems ${ }^{\mathrm{TM}}$, Abbott, Baar, Schweiz).

\section{Ergebnisse}

\section{Signalement und klinische Präsentation}

143 Signalement, klinische Symptome und Blutdruck sind in Tab. 1 und 2 144 zusammengefasst. Die Patientengruppe bestand aus 5 Europäischen 145 Kurzhaarkatzen, 1 Perser und 1 Siamesen. Die Katzen waren zwischen 7 und 17 146 Jahren alt (Median 16), 4 waren weiblich-kastriert, 3 männlich-kastriert. Die 147 häufigsten Vorstellungsgründe waren Anorexie, Schwäche, zervikale Ventroflexion 148 und Blindheit. Bei 6 Katzen wurde eine Blutdruckmessung durchgeführt, 5 der Tiere 149 wiesen eine Hypertonie auf mit einem systolischen Blutdruck zwischen 180 und 240 $150 \mathrm{mmHg}, 1$ Katze hatten einen systolischen Blutdruck von $140 \mathrm{mmHg}$. Vier Katzen mit 151 Hypertonie wiesen eine Retinaablösung auf und waren blind: bei 3 Katzen war die 152 Ablösung bilateral und bei 1 Katze unilateral mit fokalen Netzhautblutungen im kontralateralen Auge. Bei einem weiteren Tier wurden bilaterale Netzhautfalten 154 festgestellt.

\section{Hämatologie und Blutchemie}

156 Alle Katzen zeigten bei Vorstellung eine Hypokaliämie (2.2 - 3.2 mmol/l, Median 2.8, 157 Tab. 2). Eine Katze wies eine Hypernatriämie auf und bei 3 Katzen lag eine Azotämie 158 vor. Die Kreatin-Kinase (CK) war bei allen 3 Katzen, bei denen sie gemessen wurde, 159 deutlich erhöht.

\section{Ultrasonographische Untersuchung der Nebennieren}

161 Bei 6 Katzen wurde eine unilaterale Adrenomegalie festgestellt (Tab. 3). Die 162 vergrösserten Nebennieren zeigten bei 3 Katzen eine kugelige (Fig. 2) und bei 1 163 Katze eine rundlich-gelappte Form; sie wiesen bei 4 Katzen eine heterogene und bei 1642 Tieren eine homogene Echogenität auf. Bei keinem Tier gab es Hinweise auf einen 
165 Gefässeinbruch der veränderten Nebenniere. Bei 1 Katze wurden die Nebennieren

166 als ultrasonographisch normal beurteilt.

\section{Endokrinologische Untersuchungen}

169 Die Aldosteron-Konzentration lag zwischen 670 und 7853 pmol/l (Median 590); sie 170 war bei 4 Katzen erhöht, bei 1 Katze im oberen und bei 2 Katzen mit mittleren 171 Normalbereich (Tab. 2). Das Aldosteron:Renin Verhältnis bei Katze 6 betrug 29.4 172 und war damit zehnmal höher als dasjenige der Kontrolltiere (1.9 - 3.5). Im 173 Fludrocortison-Suppressionstest zeigte die Katze ein deutlich erhöhtes basales 174 Aldosteron:Kreatinin Verhältnis im Urin $\left(1600 \times 10^{-9}\right.$, Ref.: < $\left.46 \times 10^{-9}\right)$ ohne 175 nennenswerte Suppression nach Fludrocortison $\left(1200 \times 10^{-9}\right.$, Ref.: $<6 \times 10^{-9}$, 176 Djajadiningrat-Laanen et al., 2008).

\section{Behandlung und Verlauf}

178 Alle Katzen wurden medikamentös behandelt, bei 5 Katzen wurde nach 179 medikamentöser Stabilisierung eine unilaterale Adrenalektomie durchgeführt (Tab. 180 3). Die medikamentöse Therapie beinhaltete Infusionstherapie, parenterale (KCl, 20$18160 \mathrm{mmol} / \mathrm{l}$ in Ringer-Laktat als Dauerinfusion i.v.) oder orale Kaliumsubstitution 182 (Kaliumglukonat, 2 - $6 \mathrm{mmol} /$ Katze 2x täglich) und Spironolakton (Aldactone®, 5 18312.5 mg/Katze 1 - 2x täglich p.o.). Die 4 Katzen mit einem systolischen Blutdruck > $184180 \mathrm{mmHg}$ erhielten zusätzlich Amlodipine (Norvasc $\AA$ ), 0.625 - $1.25 \mathrm{mg} /$ Katze 1x 185 täglich p.o.), worauf der Blutdruck auf 140 - $170 \mathrm{~mm} \mathrm{Hg}$ abfiel; bei zwei dieser Katzen konnte das Amlodipine nach Adrenalektomie abgesetzt werden. Katze 7 mit einem systolischen Blutdruck von $180 \mathrm{mmHg}$ wurde vor Adrenalektomie nicht antihypertensiv behandelt. Die 3 azotämischen Katzen erhielten Benazepril (Fortekor®, 1.25 - 2.5 mg/Katze 1x oder 2x täglich p.o.). Eine Katze (Nr. 2) wurde wegen einer fortgeschrittenen hypertrophen Kardiomyopathie mit Furosemid (Lasix®, $2 \mathrm{mg} / \mathrm{kg} 2 x$ täglich p.o.) behandelt.

Alle Adrenalektomien verliefen komplikationslos und führten bei allen 5 Katzen zu einer Normalisierung der Elektrolytwerte. Der Blutdruck normalisierte sich nach Adrenalektomie bei 3 Katzen und blieb erhöht bei 1 Katze; die persistierende Hypertonie wurde mit einer chronischen Niereninsuffizienz erklärt. Bei 1 Katze wurde der Blutdruck nach Adrenalektomie nicht mehr bestimmt. Eine erneute

197 Ultraschalluntersuchung wurde bei 2 Katzen 1 bzw. 5 Monate nach Adrenalektomie 198 durchgeführt und zeigte eine unveränderte Grösse der verbliebenen Nebenniere; bei 
199

einer dieser Katzen wurde jedoch eine $1.3 \times 2.4 \mathrm{~cm}$ grosse, hypoechogene Masse mit hyperechogenem Zentrum im Bereich der entfernten Nebenniere festgestellt. Aufgrund der zu diesem Zeitpunkt normalen Kalium- und Aldosteron-Konzentrationen im Blut erschien ein Rezidiv des Aldosteronoms unwahrscheinlich, und es bestand der Verdacht auf ein Granulom oder Abszess. Der Besitzer lehnte einen erneuten operativen Eingriff ab.

Die Überlebenszeit ist für 4 Katzen bekannt und beträgt 3-23 Monate (Tab. 3). Für 2 Katzen (Nr. 4 und 5) liegen Verlaufsinformationen bis 7 Monate bzw. 5 Wochen nach Vorstellung vor. Eine Katze (Nr. 7) ist zum Zeitpunkt des Schreibens des Manuskripts (7 Monate nach Vorstellung) noch am Leben.

Histologische Untersuchung. In 4 Fällen lag ein Nebennierenrindenadenom und in 2 Fällen ein Nebennierenrindenkarzinom vor (Tab. 3, Fig. 3). Bei einer Katze (Nr. 1) handelte es sich um eine bilateral knotiger Hyperplasie der Nebennierenrinde; bei diesem Tier war die ultrasonographische Untersuchung der Nebennieren unauffällig verlaufen.

\section{Diskussion}

Der primäre Hyperaldosteronismus ist beim Menschen, insbesondere bei Patienten mit Hypertonie, ein gut bekanntes Krankheitsbild. In der Veterinärmedizin erlangte diese Erkrankung erst in den letzten Jahren Beachtung. Die vorliegende Fallserie zeigt, dass primärer Hyperaldosteronismus auch bei Katzen in der Schweiz vorkommt. Dabei handelte es sich mit Ausnahme einer Katze um alte Tiere $(\geq 15$ Jahre alt). Auch die bisher beschriebenen Fälle umfassten vor allem alte Katzen, die Alterspanne der 36 publizierten Fälle beträgt 5 - 18 Jahre (Eger et al., 1983, Flood et al., 1999, Mackay et al., 1999, Moore et al., 2000, Rijnberk et al., 2001, Ash et al., 2005, DeClue et al., 2005, Javadi et al., 2005, Reimer et al., 2005, Rose et al., 2007, Djajadiningrat-Laanen et al., 2008, Briscoe et al., 2009, Renschler and Dean, 2009). Die häufigsten klinischen Symptome sind Anorexie, Muskelschwäche, zervikale Ventroflexion und Blindheit. Während Anorexie und Muskelschwäche bei alten Katzen sehr unspezifische Symptome darstellen, sollte bei plötzlicher Blindheit immer eine Hypertonie ausgeschlossen werden. Hypertonie stellt eine der häufigsten Ursachen für Retinaablösung bei der Katze dar (Maggio et al., 2000), und primärer Hyperaldosteronismus ging bei 30 der 36 zuvor publizierten Fälle mit Hypertonie 
231 einher. In der vorliegenden Fallserie wiesen 5 Katzen eine Hypertonie auf, wovon 4 232 Katzen eine Retinaablösung und irreversible Blindheit zeigten.

233 Eine Hypokaliämie stellt die häufigste Blutveränderung bei Katzen mit primärem 234 Hyperaldosteronismus dar (Schulman, 2010). Auch alle Tiere in der vorliegenden 235 Fallserie zeigten einen erniedrigten Kaliumspiegel. Andere häufige Ursachen für 236 Hypokaliämie sind länger bestehende Anorexie, chronischer Durchfall und Polyurie, 237 insbesondere infolge chronischer Niereninsuffizienz. Die Unterscheidung eines 238 primären Hyperaldosteronismus von einer chronischer Niereninsuffizienz kann 239 schwierig sein: beider Erkrankungen treten vor allem bei älteren Tieren auf und 240 können mit Hypertonie, Hypokaliämie und Muskelschwäche einhergehen. Eine 241 zusätzliche Schwierigkeit für die Differenzierung der beiden Erkrankungen ergibt sich 242 aus der Tatsache, dass ca. 60\% der Katzen mit primärem Hyperaldosteronismus 243 zum Zeitpunkt der Vorstellung eine Azotämie entwickelt haben. Eine kürzlich 244 publizierte Studie postuliert, dass primärer Hyperaldosteronismus eine Rolle bei der 245 Entstehung einer chronischen Niereninsuffizienz spielt (Javadi et al., 2005). 246 Pathophysiologisch kann dies mit dem erhöhten Aldosteronspiegel und der 247 Hypertonie bei diesen Patienten erklärt werden, was zu fibrotischen und 248 sklerotischen Gefässveränderungen und damit zu Nierenschädigungen führen kann 249 (Javadi et al., 2005).

250 Hilfreich zur Diagnose eines primären Hyperaldosteronismus ist der Nachweis einer 251 Nebennierenmasse: bei 6 Katzen der vorliegenden Serie wurde eine unilaterale 252 Adrenomegalie festgestellt. Wenn das Vorliegen einer Adrenomegalie zusammen mit 253 einer persistierenden Hypokaliämie den Verdacht eines primären 254 Hyperaldosteronismus nahelegt, sollte die Diagnose mittels Hormonmessungen 255 bestätigt werden. Die Messung der Aldosteron-Konzentration im Blut stellt die 256 einfachste Hormonbestimmung dar und wird von kommerziellen Labors angeboten. 257 Es gilt zu beachten, dass die Referenzwerte je nach Labor und angewandtem Assay 258 unterschiedlich sind. Eine erhöhte Aldosteron-Konzentration bei einer Katze mit 259 persistierender Hypokaliämie und Adrenomegalie macht die Diagnose eines 260 primären Hyperaldosteronismus sehr wahrscheinlich. Die Aldosteron-Konzentration 261 wird jedoch durch viele Faktoren beeinflusst. Dehydratation erhöht die Aldosteron262 Konzentration, deshalb sollte dieser Wert bei rehydrierten Tieren bestimmt werden. 263 Ausserdem muss die Aldosteron-Konzentration in Relation zur Kaliumkonzentration 264 gesetzt werden: ein Aldosteronwert im Referenzbereich ist beim gleichzeitigen 
265 Vorliegen einer ausgeprägten Hypokaliämie als inadäquat hoch zu beurteilen, da die 266 Hypokaliämie die Aldosteronausschüttung aus den Nebennieren bremst (Fig. 1). In 267 der vorliegenden Fallserie lag die Aldosteron-Konzentration bei 4 Katzen über und 268 bei 3 Katzen im Referenzbereich.

269 Aufgrund der Problematik bei der Interpretation eines einzelnen Hormonwertes wird 270 in der Endokrinologie auf die Messung von Hormonpaaren zurückgegriffen. Dieses 271 Prinzip findet auch beim primären Hyperaldosteronismus in Form des 272 Aldosteron:Renin Quotienten Anwendung. Während unter physiologischen 273 Bedingungen ein Anstieg der Aldosteron-Konzentration von einer hohen 274 Reninaktivität begleitet wird (Fig. 1), findet beim primären Hyperaldosteronismus die 275 Aldosteronsekretion autonom, also unabhängig von der Reninkonzentration statt. 276 Deshalb ist der Quotient (Aldosteron:Renin) bei Katzen mit primärem 277 Hyperaldosteronismus höher als bei Katzen ohne dieses Krankheitsbild (Javadi et 278 al., 2005). Jedoch müssen Proben zur Messung des Renins gekühlt entnommen und 279 auf Trockeneis versandt werden und die Bestimmung kann nur in spezialisierten 280 Labors mittels enzymatischer Assays durchgeführt werden. Alternativ können zum 281 Nachweis von hormonellen Überfunktionen Suppressionstests durchgeführt werden. 282 Der Fludrocortison-Suppressionstest basiert darauf, dass es beim primären 283 Hyperaldosteronismus zu einer autonomen Aldosteronsekretion kommt, die durch 284 Fludrocortison nicht supprimiert wird. Der Test setzt eine 4-tägige Tablettengabe 285 durch den Besitzer voraus und ist beim Vorliegen einer ausgeprägten Hypokaliämie kontraindiziert. Bisher wurden Resultate erst für gesunde Katzen und eine Katze mit 287 primärem Hyperaldosteronismus publiziert (Djajadiningrat-Laanen et al., 2008). 288 Aufgrund der Schwierigkeiten der Reninmessung und der Durchführung des 289 Fludrocortison-Suppressionstests wurden diese Untersuchungen nur bei Katze 6 290 dieser Fallserie angewandt: wie erwartet zeigte sie einen 10-fach höheren 291 Aldosteron:Renin Quotienten als die gesunden Katzen und keine Suppression des 292 Urin Aldosteron: Kreatinin Verhältnisses nach Fludrocortison.

293 Kürzlich wurden 11 Katzen mit primärem Hyperaldosteronismus beschrieben, welche 294 keine vergrösserten Nebennieren und eine knotige Hyperplasie der 295 Nebennierenrinde aufwiesen (Javadi et al., 2005), die gleichen Befunde wie Katze 1 296 in dieser Fallserie. Dies verdeutlicht, dass das Vorliegen einer unilateralen 297 Adrenomegalie die Diagnose zwar unterstützt, normale Nebennieren einen primären 
298 Hyperaldosteronismus jedoch nicht ausschliessen. Bei diesen Fällen muss für die 299 Diagnose zwingend auf verschiedene Hormonmessungen zurückgegriffen werden.

300 Zusammenfassend kann festgehalten werden, dass der primäre 301 Hyperaldosteronismus eine wichtige Differentialdiagnose bei Katzen mit Schwäche, 302 Retinopathie, Hypertonie und persistierender Hypokaliämie darstellt. Eine erste 303 Verdachtsdiagnose kann anhand des Vorliegens einer Hypokaliämie, Hypertonie und 304 Nebennierenmasse gestellt werden; die definitive Diagnose basiert auf 305 verschiedenen Hormonmessungen. Begleiterkrankungen wie chronische 306 Niereninsuffizienz oder Kardiomyopathie sind häufig. Grundsätzlich kann die 307 Prognose von Katzen mit primärem Hyperaldosteronismus als verhältnismässig 308 günstig bezeichnet werden, insbesondere nach Adrenalektomie.

\section{Dank}

310 Wir danken der Abteilung für Bildgebende Diagnostik der Vetsuisse-Fakultät, 311 Universität Zürich, für das Bereitstellen der Abbildungen der ultrasonographischen 312 Untersuchung.

\section{Literaturverzeichnis}

314 1. Ash, R. A., Harvey, A. M., Tasker, S.: Primary hyperaldosteronism in the cat: a series of 13 cases. J. Feline Med. Surg. 2005, 7: 173-182.

2. Briscoe, K., Barrs, V. R., Foster, D. F., Beatty, J. A.: Hyperaldosteronism and hyperprogesteronism in a cat. J. Feline Med. Surg. 2009, 11: 758-762.

3. Brown, S., Atkins, C., Bagley, R., Carr, A., Cowgill, L., Davidson, M., Egner, B., Elliott, J., Henik, R., Labato, M., Littman, M., Polzin, D., Ross, L., Snyder, P., Stepien, R.: Guidelines for the Identification, Evaluation, and Management of Systemic Hypertension in Dogs and Cats. J. Vet. Intern. Med. 2007, 21: 542558.

4. DeClue, A. E., Breshears, L. A., Pardo, I. D., Kerl, M. E., Perlis, J., Cohn, L. A.: Hyperaldosteronism and hyperprogesteronism in a cat with an adrenal cortical carcinoma. J. Vet. Intern. Med. 2005, 19: 355-358.

5. Djajadiningrat-Laanen, S. C., Galac, S., Cammelbeeck, S. E., van Laar, K. J., Boer, P., Kooistra, H. S.: Urinary aldosterone to creatinine ratio in cats before and after suppression with salt or fludrocortisone acetate. J. Vet. Intern. Med. 2008, 22: 1283-1288. 
6. Eger, C., Robinson, W., Huxtable, C.: Primary aldosteronism (Conn's syndrome) in a cat; a case report and review of comparative aspects. J. Small Anim. Pract. 1983, 24: 293 - 307.

7. Flood, S. M., Randolph, J. F., Gelzer, A. R., Refsal, K.: Primary hyperaldosteronism in two cats. J. Am. Anim. Hosp. Assoc. 1999, 35: 411-416.

8. Javadi, S., Djajadiningrat-Laanen, S. C., Kooistra, H. S., van Dongen, A. M., Voorhout, G., van Sluijs, F. J., van den Ingh, T. S., Boer, W. H., Rijnberk, A.: Primary hyperaldosteronism, a mediator of progressive renal disease in cats. Domest. Anim. Endocrinol. 2005, 28: 85-104.

9. Javadi, S., Slingerland, L. I., van de Beek, M. G., Boer, P., Boer, W. H., Mol, J. A., Rijnberk, A., Kooistra, H. S.: Plasma renin activity and plasma concentrations of aldosterone, cortisol, adrenocorticotropic hormone, and alpha-melanocytestimulating hormone in healthy cats. J. Vet. Intern. Med. 2004, 18: 625-631.

10. Jepson, R. E.: Feline systemic hypertension: Classification and pathogenesis. J. Feline Med. Surg. 2011, 13: 25-34.

11. MacKay, A. D., Holt, P. E., Sparkes, A. H.: Successful surgical treatment of a cat with primary aldosteronism. J. Feline Med. Surg. 1999, 1: 117-122.

12. Maggio, F., DeFrancesco, T. C., Atkins, C. E., Pizzirani, S., Gilger, B. C., Davidson, M. G.: Ocular lesions associated with systemic hypertension in cats: 69 cases (1985-1998). J. Am. Vet. Med. Assoc. 2000, 217: 695-702.

13. Moore, L. E., Biller, D. S., Smith, T. A.: Use of abdominal ultrasonography in the diagnosis of primary hyperaldosteronism in a cat. J. Am. Vet. Med. Assoc. 2000, 217: 213-215, 197.

14. Nussberger, J., Fasanella d'Amore, T., Porchet, M., Waeber, B., Brunner, D. B., Brunner, H. R., Kler, L., Brown, A. N., Francis, R. J.: Repeated administration of the converting enzyme inhibitor cilazapril to normal volunteers. J. Cardiovasc. Pharmacol. 1987, 9: 39-44.

15. Nussberger, J., Waeber, B., Brunner, H. R., Burris, J. F., Vetter, W.: Highly sensitive microassay for aldosterone in unextracted plasma: comparison with two other methods. J. Lab. Clin. Med. 1984, 104: 789-796.

16. Reimer, S. B., Pelosi, A., Frank, J. D., Steficek, B. A., Kiupel, M., Hauptman, J. G.: Multiple endocrine neoplasia type I in a cat. J. Am. Vet. Med. Assoc. 2005, 227: 101-104, 186. 
17. Renschler, J. S., Dean, G. A.: What is your diagnosis? Abdominal mass aspirate in a cat with an increased Na:K ratio. Vet. Clin. Pathol. 2009, 38: 69-72.

18. Reusch, C. E., Schellenberg, S., Wenger, M.: Endocrine hypertension in small animals. Vet. Clin. North Am. Small Anim. Pract. 2010, 40: 335-352.

19. Rijnberk, A., Voorhout, G., Kooistra, H. S., van der Waarden, R. J., van Sluijs, F. J., Boer, P., Boer, W. H.: Hyperaldosteronism in a cat with metastasised adrenocortical tumour. Vet. Q. 2001, 23: 38-43.

20. Rose, S. A., Kyles, A. E., Labelle, P., Pypendop, B. H., Mattu, J. S., Foreman, O., Rodriguez, C. O., Jr., Nelson, R. W.: Adrenalectomy and caval thrombectomy in a cat with primary hyperaldosteronism. J. Am. Anim. Hosp. Assoc. 2007, 43: 209-214.

21. Schulman, R. L.: Feline primary hyperaldosteronism. Vet. Clin. North Am. Small Anim. Pract. 2010, 40: 353-359.

22. Zimmer, C., Horauf, A., Reusch, C.: Ultrasonographic examination of the adrenal gland and evaluation of the hypophyseal-adrenal axis in 20 cats. J. Small Anim. Pract. 2000, 41: 156-160.

Korrespondenz: Prof. Dr. Claudia Reusch, Klinik für Kleintiermedizin, VetsuisseFakultät der Universität Zürich, Winterthurerstrasse 260, 8057 Zürich. creusch@vetclinics.uzh.ch 


\section{Legenden}

385 Fig. 1. Schematische Darstellung des Renin-Angiotensin-Aldosteron Symstems und 386 Auswirkungen eines inadäquat hohen Aldosteronspiegels auf den Elektrolyt-, Säure387 Base- und Wasserhaushalt. Renin wird bei einem Abfall des zirkulierenden 388 Blutvolumens oder der Nierendurchblutung aus dem juxtaglomerulären Apparat der 389 Niere ausgeschüttet. Renin wirkt als Protease auf Angiotensinogen, welches in der 390 Leber produziert wird, und wandelt es in Angiotensin I um. Angiotensin I wird durch 391 das Angiotensin-Converting Enzym (ACE) in Angiotensin II konvertiert. Angiotensin II 392 bewirkt, nebst seiner Rolle als potenter Vasokonstriktor, eine Ausschüttung von 393 Aldosteron aus der Zona glomerulosa der Nebennierenrinde. Eine Hyperkaliämie 394 andererseits hemmt die Aldosteronsekretion. Aldosteron wirkt am distalen Tubulus 395 und Sammelrohr der Niere: es induziert eine Natrium- und Wasserreabsorption und 396 eine Kalium- und $\mathrm{H}^{+}$- Sekretion. Ein inadäquater hoher Aldosteronspiegel, als Folge 397 eines primären Hyperaldosteronismus, führt zu einer erhöhten Wasserreabsorption 398 und einem erhöhten Kalium- und $\mathrm{H}^{+}$-Verlust. Die Folgen sind Hypertonie, 399 Hypokaliämie und metabolische Alkalose.

400 Fig. 2. Ultrasonographische Darstellung der rechtsseitigen Nebennierenmasse (a.) 401 und der unauffälligen linken Nebenniere (b.) von Katze 6.

402 Fig. 3. Histopathologische Untersuchung eines Nebennierenrindenadenoms und 403 karzinoms von Katze 6 und 4. a. Nebennierenrindenadenom: durch feine 404 Bindegewebekapsel abgegrenztes Proliferat aus Nenbennierenrindenzellen. In 405 der oberen linken Bildhälfte sind Anteile des nicht-neoplastischen Nebennierenmarks 406 sichtbar. b. Nebennierenrindenkarzinom: infiltrativ wachsendes, solides Proliferat von 407 Nebennierenrindenzellen. 
Fig. 1

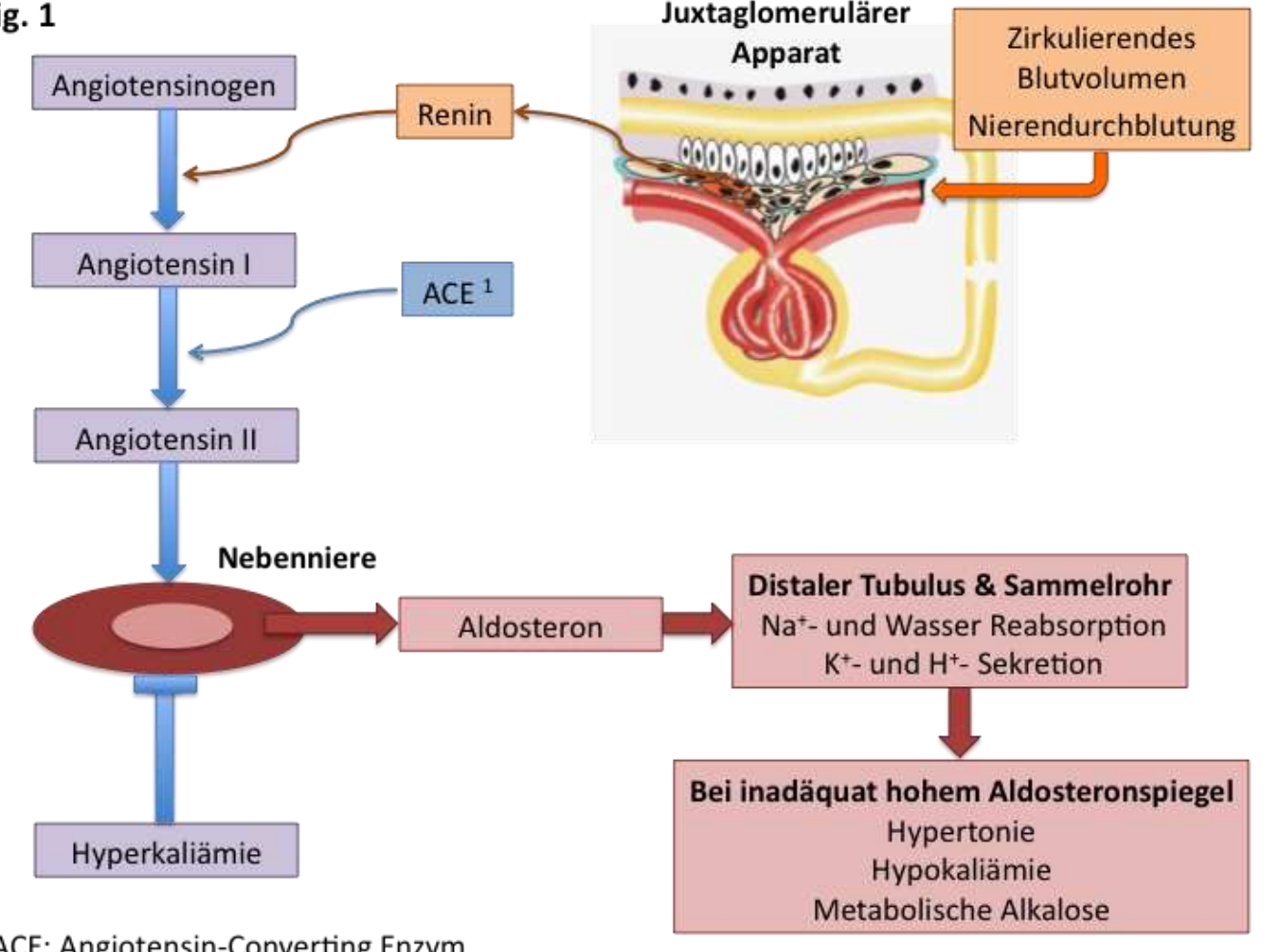

${ }^{1}$ ACE: Angiotensin-Converting Enzym

Fig. 2

a.

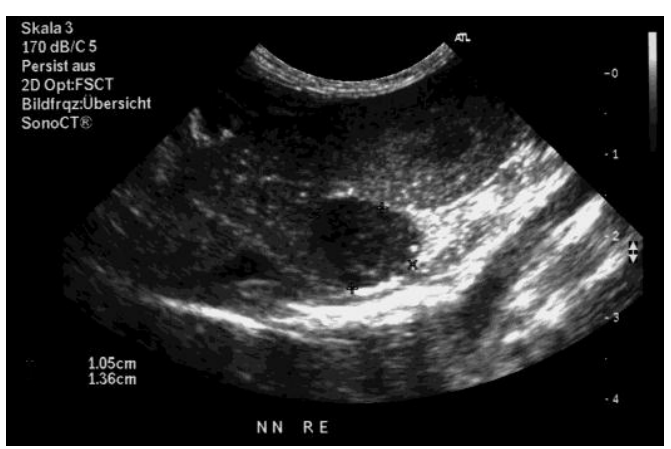

Fig. 3

a.

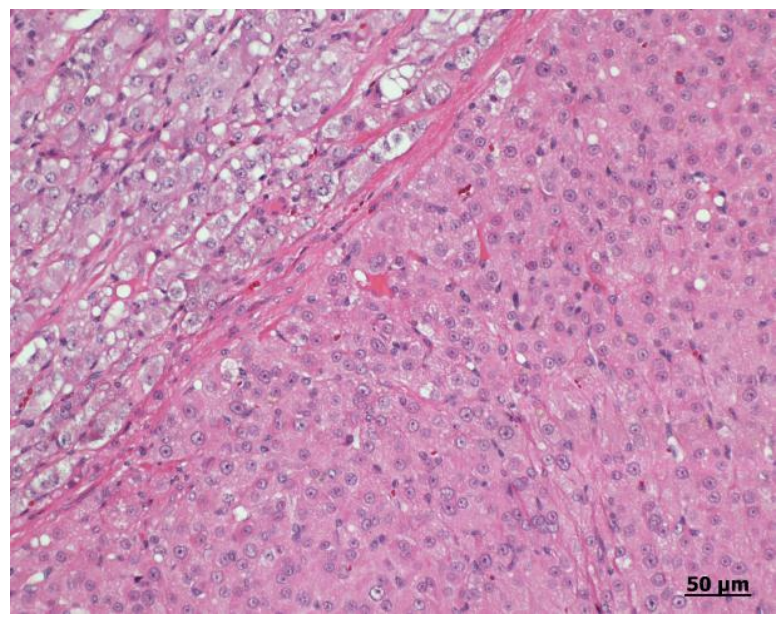

b.

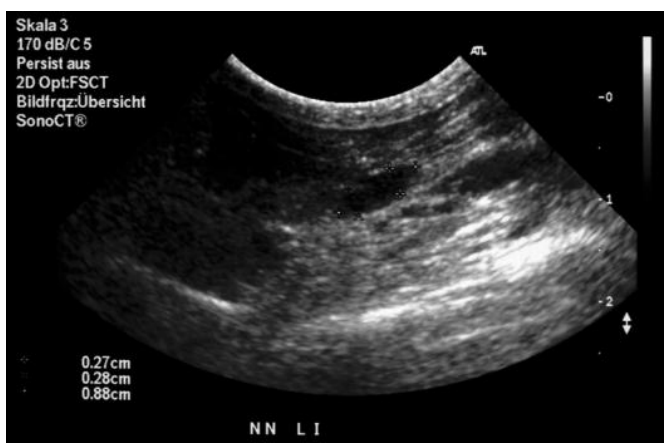

b.

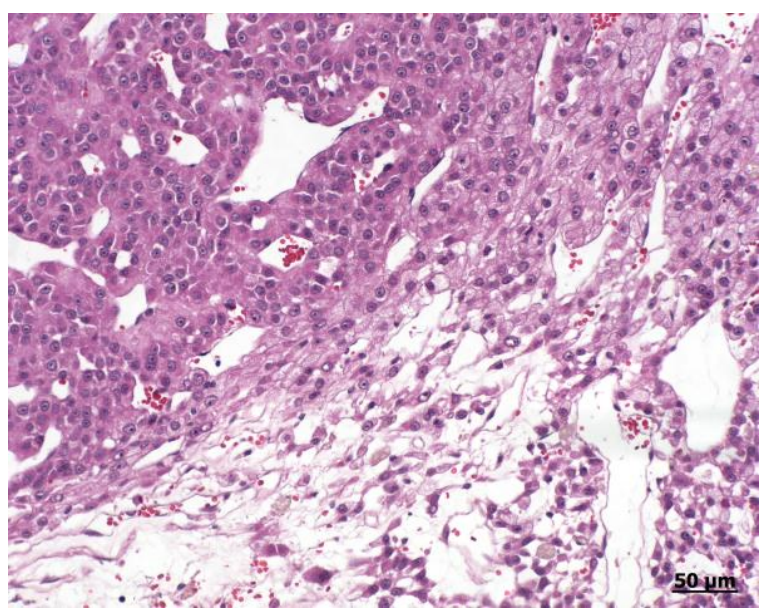


Tabelle 1. Signalement und klinische Symptome von 7 Katzen mit primärem Hyperaldosteronismus.

\begin{tabular}{|c|c|c|c|c|c|c|c|c|}
\hline \multirow[b]{2}{*}{ Katze } & \multirow[b]{2}{*}{ Rasse $^{1}$} & \multirow[b]{2}{*}{$\begin{array}{l}\text { Alter } \\
\text { (Jahre) }\end{array}$} & \multirow[b]{2}{*}{ Geschlecht $^{2}$} & \multicolumn{5}{|c|}{ Klinische Präsentation } \\
\hline & & & & Anorexie & Schwäche & $\begin{array}{c}\text { Zervikale } \\
\text { Ventroflexion }\end{array}$ & Blindheit & $\begin{array}{l}\text { Netzhaut- } \\
\text { ablösung }\end{array}$ \\
\hline 1 & Perser & 17 & wk & $\mathrm{Ja}$ & $\mathrm{Ja}$ & Nein & $\mathrm{Ja}$ & $\mathrm{Ja}$ \\
\hline 2 & $\mathrm{EKH}$ & 7 & $\mathrm{mk}$ & Nein & $\mathrm{Ja}$ & $\mathrm{Ja}$ & Nein & $\begin{array}{l}\text { Netzhaut- } \\
\text { falten }\end{array}$ \\
\hline 3 & $\mathrm{EKH}$ & 15 & wk & $\mathrm{Ja}$ & Nein & $\mathrm{Ja}$ & Nein & n.d. ${ }^{3}$ \\
\hline 4 & $\mathrm{EKH}$ & 15 & wk & $\mathrm{Ja}$ & $\mathrm{Ja}$ & Nein & $\mathrm{Ja}$ & $\mathrm{Ja}$ \\
\hline 5 & $\mathrm{EKH}$ & 16 & $\mathrm{mk}$ & $\mathrm{Ja}$ & Nein & Nein & $\mathrm{Ja}$ & $\mathrm{Ja}$ \\
\hline 6 & $\mathrm{EKH}$ & unbekannt & wk & Nein & $\mathrm{Ja}$ & Nein & $\mathrm{Ja}$ & $\mathrm{Ja}$ \\
\hline 7 & Siamese & 16 & $\mathrm{mk}$ & Nein & $\mathrm{Ja}$ & Nein & Nein & n.d. ${ }^{3}$ \\
\hline Total & $\begin{array}{c}5 \text { EKH, } \\
1 \text { Perser, } \\
1 \text { Siamese }\end{array}$ & & $4 \mathrm{wk}, 3 \mathrm{mk}$ & $4 / 7$ & $5 / 7$ & $2 / 7$ & $4 / 7$ & $4 / 7$ \\
\hline
\end{tabular}

${ }^{1}$ EKH, Europäische Kurzhaarkatze, ${ }^{2}$ wk, weiblich-kastriert; mk, männlich-kastriert, ${ }^{3}$ n.d., ophtalmologische Untersuchung nicht durchgeführt 
Tabelle 2. Blutdruck, blutchemische Ergebnisse und Serum Aldosteron-Konzentration bei 7 Katzen mit primärem Hyperaldosteronismus.

\begin{tabular}{lccccccc}
\hline Katze & $\begin{array}{c}\text { Blutdruck } \\
(\mathrm{mm} \mathrm{Hg})\end{array}$ & $\begin{array}{c}\text { Kalium } \\
(\mathrm{mmo} / \mathrm{l})\end{array}$ & $\begin{array}{c}\text { Natrium } \\
(\mathrm{mmol} / \mathrm{l})\end{array}$ & $\begin{array}{c}\text { Harnstoff } \\
(\mathrm{mmol} / \mathrm{l})\end{array}$ & $\begin{array}{c}\text { Kreatinin } \\
(\mathrm{umol} / \mathrm{l})\end{array}$ & $\begin{array}{c}\text { CK } \\
(\mathrm{U} / \mathrm{l})\end{array}$ & $\begin{array}{c}\text { Aldosteron } \\
(\mathrm{pmol} / \mathrm{l})\end{array}$ \\
\hline Normalwerte & $<150 \mathrm{~mm} \mathrm{Hg}$ & $3.8-5.5^{1}$ & $158-165^{1}$ & $7.4-12.6^{1}$ & $98-163^{1}$ & $77-355^{1}$ & $19-1579^{1}$ \\
\hline 1 & 240 & 3.2 & 159 & 11.2 & 123 & n.d. $^{2}$ & 1238 \\
2 & n.d. $^{2}$ & 2.7 & 156 & 12.5 & 185 & 16400 & 670 \\
3 & 140 & 2.9 & 165 & 9.7 & 142 & n.d. $^{2}$ & 2737 \\
4 & 240 & 3.0 & 158 & 11.0 & 101 & 182950 & 1634 \\
5 & 240 & 2.4 & 152 & 13.7 & 201 & n.d. $^{2}$ & 706 \\
6 & 185 & 2.8 & 182 & 32.3 & 321 & n.d. ${ }^{2}$ & 3546 \\
7 & 180 & 2.2 & 161 & 11.7 & 136 & 9205 & 7853 \\
\hline \multirow{2}{*}{ Total } & erhöht & erniedrigt & erhöht & erhöht & erhöht & erhöht & erhöht \\
& $5 / 6$ & $7 / 7$ & $1 / 7$ & $2 / 7$ & $3 / 7$ & $3 / 3$ & $4 / 7$ \\
\hline
\end{tabular}

\footnotetext{
${ }^{1} 5 \%$ und $95 \%$ Perzentil, ${ }^{2}$ nicht durchgeführt
} 
Tabelle 3. Ultrasonographische und histologische Untersuchung, Therapie und Krankheitsverlauf bei 7 Katzen mit primärem Hyperaldosteronismus.

\begin{tabular}{|c|c|c|c|c|c|c|}
\hline \multirow[t]{2}{*}{ Katze } & \multicolumn{2}{|c|}{$\begin{array}{l}\text { Vergrösserte } \\
\text { Nebenniere }\end{array}$} & \multirow[t]{2}{*}{ Histologie } & \multirow{2}{*}{$\begin{array}{l}\text { Adrenal- } \\
\text { ektomie }\end{array}$} & \multirow[t]{2}{*}{ Verlauf } & \multirow[t]{2}{*}{ Überlebenszeit ${ }^{1}$} \\
\hline & links & rechts & & & & \\
\hline 1 & Nein & Nein & $\begin{array}{c}\text { Knotige } \\
\text { Hyperplasie }\end{array}$ & Nein & $\begin{array}{c}\text { Euthanasie wegen genereller } \\
\text { Verschlechterung des } \\
\text { Allgemeinzustandes }\end{array}$ & 3 Monate \\
\hline 2 & Nein & $\mathrm{Ja}$ & Adenom & Nein & $\begin{array}{c}\text { Euthanasie wegen } \\
\text { dekompensierter Kardiomyopathie }\end{array}$ & 3 Monate \\
\hline 3 & Nein & $\mathrm{Ja}$ & Adenom & $\mathrm{Ja}$ & $\begin{array}{l}\text { Euthanasie wegen multipler } \\
\text { abdominaler Massen }\end{array}$ & 23 Monate \\
\hline 4 & $\mathrm{Ja}$ & Nein & Karzinom & $\mathrm{Ja}$ & $\begin{array}{l}\text { Normale Elektrolytwerte, stabile } \\
\text { Nierenwerte, keine Hypertonie }\end{array}$ & $\begin{array}{c}\text { Nach } 7 \text { Monate keine weitere } \\
\text { Verlaufskontrolle }\end{array}$ \\
\hline 5 & Nein & $\mathrm{Ja}$ & Karzinom & $\mathrm{Ja}$ & $\begin{array}{l}\text { Normale Elektrolytwerte, stabile } \\
\text { Nierenwerte, keine Hypertonie }\end{array}$ & $\begin{array}{c}\text { Nach } 5 \text { Wochen keine weitere } \\
\text { Verlaufskontrolle }\end{array}$ \\
\hline 6 & Nein & $\mathrm{Ja}$ & Adenom & $\mathrm{Ja}$ & $\begin{array}{c}\text { Euthanasie wegen hochgradiger } \\
\text { renaler Azotämie }\end{array}$ & 3 Monate \\
\hline 7 & $\mathrm{Ja}$ & Nein & Adenom & $\mathrm{Ja}$ & $\begin{array}{l}\text { Erneut Masse im Bereich der } \\
\text { entfernten linken Nebenniere }\end{array}$ & $\begin{array}{c}\text { Zum Zeitpunkt des Schreibens des } \\
\text { Manuskripts ( } 7 \text { Monate nach } \\
\text { Vorstellung) am Leben }\end{array}$ \\
\hline
\end{tabular}

\footnotetext{
${ }^{1}$ Zeit von erster Präsentation bis zur Euthanasie oder letzten Verlaufskontrolle
} 